\title{
Ventilator-associated pneumonia in extracorporeal membrane oxygenation-assisted patients
}

\author{
Guillaume Franchineau $^{1,2}$, Charles Edouard Luyt ${ }^{1,2}$, Alain Combes ${ }^{1,2}$, Matthieu Schmidt ${ }^{1,2}$ \\ ${ }^{1}$ Sorbonne Universités, UPMC Univ Paris 06, INSERM UMRS_1166-iCAN, Institute of Cardiometabolism and Nutrition, Paris, France; \\ ${ }^{2}$ Assistance Publique-Hôpitaux de Paris, Pitié-Salpêtrière Hospital, Medical Intensive Care Unit, Paris, France \\ Contributions: (I) Conception and design: None; (II) Administrative support: None; (III) Provision of study materials or patients: None; (IV) \\ Collection and assembly of data: None; (V) Data analysis and interpretation: None; (VI) Manuscript writing: All authors; (VII) Final approval of \\ manuscript: All authors. \\ Correspondence to: Matthieu Schmidt, MD, PhD. Service de Réanimation Médicale, iCAN, Institute of Cardiometabolism and Nutrition, Hôpital de \\ la Pitié-Salpêtrière, 47, bd de l'Hôpital, 75651 Paris Cedex 13, France. Email: matthieu.schmidt@aphp.fr.
}

\begin{abstract}
Extracorporeal membrane oxygenation (ECMO) provides a circulatory and/or respiratory assistance in case of refractory cardiogenic shock or acute respiratory distress syndrome (ARDS). Due to their extreme critical illness, these patients usually require prolonged mechanical ventilation, which is an inherent risk of ventilator-associated pneumonia (VAP). Although microorganisms responsible of VAP on ECMO are similar to those found in non-ECMO patients, classical suspicion criteria of VAP are no longer relevant on ECMO. Frequent severe chest X-Ray impairments, and poor specificity of the classical biomarkers on ECMO make VAP diagnosis challenging. In addition, significant drug pharmacokinetic modifications by the device may lead to low plasmatic antibiotic concentration and potential treatment failure. Consequently, rate of treatment failure and relapse appear high in that population (up to 30\%), with significant impact on mortality and on the ECMO duration.
\end{abstract}

Keywords: Extracorporeal membrane oxygenation (ECMO); ventilator-associated pneumonia (VAP); mortality; pseudomonas aeruginosa; pharmacokinetics

Submitted Oct 04, 2018. Accepted for publication Oct 08, 2018.

doi: $10.21037 / \mathrm{atm} .2018 .10 .18$

View this article at: http://dx.doi.org/10.21037/atm.2018.10.18

Extracorporeal membrane oxygenation (ECMO) is a respiratory and circulatory support that supplies gas exchanges and provides arterial blood flow when conventional treatments have failed. Veno-venous ECMO ( VV-ECMO) is now considered as an option for patients with severe ARDS for whom conventional mechanical ventilation has failed to maintain sufficient blood oxygenation, decarboxylation, and/or low airways pressure (1). Its use has markedly increased since 2009 with the H1N1 influenza pandemic and the publication of the CESAR trial (2). Recently, the EOLIA multicenter trial has highlighted that this technic is now safe and its early use may be beneficial for severe patients for whom spontaneous evolution would have been fatal (3). On the other hand, veno-arterial ECMO (VA-ECMO) is a mechanical circulatory support that provides arterial blood flow in case of refractory cardiogenic shock. Its main uses are acute myocardial infarction, dilated cardiomyopathy, myocarditis, pulmonary embolism, dilated cardiomyopathy, or refractory cardiac arrest (4). Due to the high severity of these patients at cannulation, their frequent ECMO-related complications such as pulmonary edema, and multiple organ failure during their intensive care unit (ICU) stay, these very severe patients are more likely to have prolonged mechanical ventilation duration. Consequently, they are particularly exposed to ventilator-associated pneumonia (VAP), which may also be favored by immune paralysis common to all patients having a long ICU stay (5). 


\section{Incidence of VAP on ECMO}

Variable incidences of VAP afflicting ECMO patients were previously reported by few retrospective studies. Two of them performed in Taiwan mixed a population of $80 \%$ VA and 20\% VV ECMO between 2001 and 2007 (6,7). VAP was diagnosed in $3.5 \%$ of patients. This low rate was however not confirmed by the following studies and could be explained by the frequent use of prolonged prophylactic antibiotic during the ECMO run. More recent ones, performed between 2005 and 2011, reported a prevalence of VAP from $15.8 \%$ to $24 \%(8,9)$, with however a small population studied. The largest study to date in the field, was published by Schmidt et al. in 2012 (10). Two hundred and twenty patients who underwent VA-ECMO for more than 48 hours between 2003 and 2009 were studied. One hundred and sixty-three (74\%) developed a VAP after of $8 \pm 11$ days of mechanical ventilation. Notably, $36 \%$ of these VAP episodes were complicated by a septic shock, illustrating the potential severity of this complication. More recently, Bouglé et al. studied specifically VAP rate under VA-ECMO in 152 patients (11). Eighty-five patients (55.9\%) developed at least one VAP episode, which represents an incidence of 60.6 VAP episode per 1,000 ECMO days after 5 [3-12] days of mechanical ventilation. Lastly, no study specifically assessed the incidence of VAP in a population of $\mathrm{VV}-\mathrm{ECMO}$, which is known to have a prolonged time on mechanically ventilation (12). However, a VAP incidence of 31 episodes per 1,000 ECMO days in a population of 92 patients ( $87 \%$ VV-ECMO) was recently reported (13). In addition, higher risk of VAP in ECMO patients compared to other ICU patients was confirmed in a recent meta-analysis of Biffi et al., with an overall incidence of 24.4 episodes per 1,000 ECMO days (14). Higher severity of these patients who frequently had a SOFA score $>10$ and a SAPS $2>50$ is probably the main risk factor of VAP in this population $(10,11)$. In addition, dysfunction of alveolar macrophages (15) or a decreased monocytes response to pathogens (16) induced by extracorporeal support may also contribute to this higher risk.

\section{Causative organisms}

Micro-organisms associated with VAP on ECMO did not differ from classical epidemiology of VAP in non ECMO patients (6-9). Gram negative bacteria were involved in up $70 \%$ of VAP in VA-ECMO treated patients $(10,11)$, with Pseudomonas aeruginosa found in $18-26 \%$ of cases
$(10,11)$. Similar results were reported with VV-ECMO, were $63 \%$ of VAP were due to Gram negative bacteria, with $13 \%$ of pseudomonas aeruginosa (13). Lastly, while Gram positive microorganisms were found in $20 \%$ of VAP, a large predominance of Staphylococcus Aureus was reported $(10,11,13)$.

\section{Risk factors and impact of VAP on ECMO}

Identification of risk factors of VAP is challenging, as most of the studies reported overall risk factors associated with ECMO-related infection and not specifically VAP on ECMO. However, duration of ECMO $(6,7,14,17)$, immunocompromised status (6), and severity of patient at ECMO cannulation (i.e., SOFA or SAPS II scores) $(8,9)$ were commonly reported as associated with a higher risk of ECMO-related infections. Impact of the ECMO setting (i.e., $\mathrm{VV}$ or $\mathrm{VA})$ is unclear $(6,9,17)$, even if a higher risk of nosocomial infection was noted with ECMO for a cardiac indication (i.e., VA configuration) (14). Lastly, age seems to be an important risk factor as rates were highest in the adult $v s$. the pediatric and neonatal populations (30.6 vs. $20.8 v s$. 10.1 infections per 1,000 ECMO days, respectively) (17). In addition, older age ( $>50$ years old), ECMO for cardiogenic shock, higher SAPS II score, diagnosis of VAP, and infection by multidrug-resistant bacteria were independently associated to increased death rate in an adult population (13). To date, only Bouglé et al. focused on identifying risk factors of VAP on VA-ECMO. Risk factors for VAP in univariate analysis were: age $>65$ years old [risk ratio (RR) 1.73 ; $95 \%$ confidence interval $(\mathrm{CI}), 1.13-2.63, \mathrm{P}=0.01]$, SOFA score at admission (RR 1.09; 95\% CI, 1.02-1.17, $\mathrm{P}=0.01$ ), history of hypertension (RR 2.25; 95\% CI, 1.43-3.53, P=0.0004), active smoking (RR 1.55; 95\% CI, 0.96-2.49, $\mathrm{P}=0.07$ ) and COPD (RR 2.15; 95\% CI, 1.30-3.55, P=0.003) whereas the female gender (RR 0.44; 95\% CI, 0.24-0.81, $\mathrm{P}=0.008$ ) was protective. However, insufficient power secondary to too many missing data, precluded to perform a multivariate analysis (11).

Negative impact of ECMO-related bloodstream infections on the outcome has been consistently reported. In addition, it was also associated with a longer ICU length of stay, mechanical ventilation and ECMO duration $(13,14)$. Impact of VAP on ECMO seems to be similar. VAP was associated with longer ECMO duration [hazard ratio (HR) 1.47 (1.05 \pm 2.05$), \mathrm{P}=0.025]$ and an increased mortality (HR 3.05; 95\% CI, 1.66-5.63, P<0.001). However, VAP was not associated with increased length of mechanical ventilation 
in that study (11). The significant attributable mortality of VAP, reported up to $13 \%$ (18), should prompt physicians to focus on VAP prevention and early diagnosis.

\section{Prevention and diagnosis of VAP on ECMO}

As well demonstrated in non-ECMO patients, several simple VAP-prevention measures should also be applied in ECMO patients. Notably, endotracheal tube cuff pressure maintained between 20 and $30 \mathrm{cmH}_{2} \mathrm{O}$, oral chlorhexidine application four times per day, and semi recumbent body positioning $\left(30^{\circ}\right.$ to $\left.45^{\circ}\right)$ are mandatory.

Early diagnosis of VAP on ECMO is, however, challenging as current criteria to suspect VAP seem nonrelevant and inapplicable on ECMO. Indeed, diagnosis of VAP is commonly suspected when a patient develops new radiologic lung infiltrates plus at least two of the following items: fever greater than $38^{\circ} \mathrm{C}$, leukocytosis or leukopenia, and purulent secretions (19). Strict application of these criteria on ECMO is difficult. For instance, new radiologic lung infiltrates is difficult to interpret on ECMO as more than $30 \%$ of patients under VA-ECMO develop a pulmonary edema $(20,21)$, (i.e., common cause of hypoxemia and new radiologic lung infiltrates), without VAP. Similar limits are also applicable on VV, where radiologic lesions frequently involved the four quadrants $(2,3)$, and ultra-protective ventilation on ECMO may cause decruitment, which could exacerbate a chest X-ray aspect without VAP. "Fever greater than $38^{\circ} \mathrm{C}$ " is not a relevant criterion on ECMO as fever is frequently masked by extracorporeal circulation, inducing blood cooling. Waiting for fever to suspect VAP on ECMO could lead to a major diagnosis delay. Similarly, an increase in CRP, D-dimer, leucocytes, fibrinogen decline, or thrombocytopenia are frequent and are non-specific biological abnormalities under ECMO. For instance, systemic inflammatory response syndrome induced by extracorporeal circulation initiation could, itself, explain these biological modifications. Likewise, a decrease in fibrinogen and a thrombocytopenia on ECMO are relatively frequent and most often related to a circuit-related consumption coagulopathy. Thus, the usual markers of infection are difficult to interpret under ECMO. It seems, therefore, necessary to take into consideration the kinetics of these markers and to interpret these anomalies with regard to possible complications of the circuit. A strategy combining an awareness of the medical and nursing teams at the high risk of infection in these patients, combined with a pro-active strategy to get bacteriological samples at the slightest suspicion is, to date, a way to reduce the impact of these infections on outcome.

\section{Treatment of VAP on ECMO}

In addition to diagnosis issues, treatment of VAP on ECMO is also challenging. Bougle et al. reported a recurrence of VAP on ECMO for $37.2 \%$ of patients, with 10 persistence $(11.8 \%), 8$ relapses $(9.4 \%)$, and 19 superinfections (22.3\%) (11). Risk factors for VAP treatment failure were renal replacement therapy [HR 13.05 (1.73; 98.56), $\mathrm{P}=0.013]$ and documentation of Pseudomonas aeruginosa $[\mathrm{HR}$ 2.36 (1.04; 5.35), $\mathrm{P}=0.04]$.

Most of the common drugs daily used in ICU, including antibiotic, have altered pharmacokinetics with ECMO, which could lead to poor achievement of targeted concentrations, inducing potential treatment failure with persistence or relapse (22-24). Potential factors affecting drug disposition in critically ill patients have been extensively studied. Main ones are hemodynamic instability, concurrent drug therapy, mechanical ventilation, nutrition support, concurrent disease states, proteinbinding alterations or, endogenous cytokine release. In addition, it is worth stressing the dynamic nature of these factors which may change overtime. The large surface of tubing and membrane could lead to drug sequestration, and therefore induce an increase of the volume of distribution (Vd). Major risk factors of drugs sequestration are: (I) drug properties such as molecular size, plasma protein binding or lipophilicity (25); (II) age of the circuit (26) or; (III) type of the membrane (27). Others factors may contribute to increase the $\mathrm{Vd}$ on ECMO. As reported with nonECMO patients, high systemic inflammation level and severe organ failure are major risk factors, whereas large fluid administration and frequent transfusions may also induce hemodilution. Lastly, frequent acute kidney injury on ECMO may also play a key role in the modifications of pharmacokinetics in this population. To date, there is an urgent need to fully elucidate the behaviors of drugs during ECMO with clinical population-based pharmacokinetics studies. In the interim, frequent antibiotics dosing on ECMO is recommended until robust dosing guidelines become available.

An upcoming challenge for VAP prevention will be to likely reduce mechanical ventilation on ECMO. The concept of "awake ECMO" has been developed a decade 
ago, and seems promising in the context of VA-ECMO and acute on chronic respiratory failure $(28,29)$. In the context of VV-ECMO for severe ARDS, early extubation on ECMO appears more complex, as it is difficult to control the intense central respiratory drive of these patients. Nevertheless, it is worth noting that this strategy may be feasible for some patients (i.e., immunocompromised patients), and few centers have starting to develop this strategy $(28,29)$.

\section{Conclusions}

ECMO-assisted patients are likely exposed to VAP, with a higher incidence reported in this population compared to non-ECMO ICU patients. Consequently, VAP on ECMO is associated with an increased mortality and a longer ICU length of stay. Although microorganisms involved in VAP on ECMO are similar than classical epidemiology of ventilated ICU patients, the diagnostic and the treatment are both challenging. It could explain the high recurrence of VAP in this population. Future research is urgently needed on antibiotics pharmacokinetics on ECMO. In the interim, frequent dosing is recommended until robust dosing guidelines become available.

\section{Acknowledgements}

None.

\section{Footnote}

Conflicts of Interest: The authors have no conflicts of interest to declare.

\section{References}

1. Brodie D, Bacchetta M. Extracorporeal Membrane Oxygenation for ARDS in Adults. N Engl J Med 2011;365:1905-14.

2. Peek GJ, Mugford M, Tiruvoipati R, et al. Efficacy and economic assessment of conventional ventilatory support versus extracorporeal membrane oxygenation for severe adult respiratory failure (CESAR): a multicentre randomised controlled trial. Lancet 2009;374:1351-63.

3. Combes A, Hajage D, Capellier G, et al. Extracorporeal Membrane Oxygenation for Severe Acute Respiratory Distress Syndrome. N Engl J Med 2018;378:1965-75.

4. Abrams D, Garan AR, Abdelbary A, et al. Position paper for the organization of ECMO programs for cardiac failure in adults. Intensive Care Med 2018;44:717-29.

5. Hotchkiss RS, Monneret G, Payen D. Sepsis-induced immunosuppression: from cellular dysfunctions to immunotherapy. Nat Rev Immunol 2013;13:862-74.

6. Sun HY, Ko WJ, Tsai PR, et al. Infections occurring during extracorporeal membrane oxygenation use in adult patients. J Thorac Cardiovasc Surg 2010;140:1125-32.e2.

7. Hsu MS, Chiu KM, Huang YT, et al. Risk factors for nosocomial infection during extracorporeal membrane oxygenation. J Hosp Infect 2009;73:210-6.

8. Pieri M, Agracheva N, Fumagalli L, et al. Infections occurring in adult patients receiving mechanical circulatory support: The two-year experience of an Italian National Referral Tertiary Care Center. Med Intensiva 2013;37:468-75.

9. Aubron C, Cheng AC, Pilcher D, et al. Infections Acquired by Adults Who Receive Extracorporeal Membrane Oxygenation Risk Factors and Outcome. Infect Control Hosp Epidemiol 2013;34:24-30.

10. Schmidt M, Bréchot N, Hariri S, et al. Nosocomial infections in adult cardiogenic shock patients supported by venoarterial extracorporeal membrane oxygenation. Clin Infect Dis 2012;55:1633-41.

11. Bouglé A, Bombled C, Margetis D, et al. Ventilatorassociated pneumonia in patients assisted by venoarterial extracorporeal membrane oxygenation support: Epidemiology and risk factors of treatment failure. PLoS One 2018;13:e0194976.

12. Schmidt M, Zogheib E, Rozé H, et al. The PRESERVE mortality risk score and analysis of long-term outcomes after extracorporeal membrane oxygenation for severe acute respiratory distress syndrome. Intensive Care Med 2013;39:1704-13.

13. Grasselli G, Scaravilli V, Di Bella S, et al. Nosocomial Infections During Extracorporeal Membrane Oxygenation: Incidence, Etiology, and Impact on Patients' Outcome. Crit Care Med 2017;45:1726-33.

14. Biffi S, Di Bella S, Scaravilli V, et al. Infections during extracorporeal membrane oxygenation: epidemiology, risk factors, pathogenesis and prevention. Int J Antimicrob Agents 2017;50:9-16.

15. Chalk K, Meisel C, Spies C, et al. Dysfunction of alveolar macrophages after 3 cardiac surgery and postoperative pneumonia? - an 5 observational study. Crit Care 2013;17:R285.

16. Hadley JS, Wang JE, Michaels LC, et al. Alterations in inflammatory capacity and TLR expression on monocytes 
and neutrophils after cardiopulmonary bypass. Shock 2007;27:466-73.

17. Bizzarro MJ, Conrad SA, Kaufman DA, et al. Infections acquired during extracorporeal membrane oxygenation in neonates, children, and adults. Pediatr Crit Care Med 2011;12:277-81.

18. Melsen WG, Rovers MM, Groenwold RH, et al. Attributable mortality of ventilator-associated pneumonia: a meta-analysis of individual patient data from randomised prevention studies. The Lancet Infectious Diseases 2013;13:665-71.

19. American Thoracic Society; Infectious Diseases Society of America. Guidelines for the Management of Adults with Hospital-acquired, Ventilator-associated, and Healthcareassociated Pneumonia. Am J Respir Crit Care Med 2005;171:388-416.

20. Demondion P, Fournel L, Golmard JL, et al. Predictors of 30-day mortality and outcome in cases of myocardial infarction with cardiogenic shock treated by extracorporeal life support. Eur J Cardiothorac Surg 2014;45:47-54.

21. Combes A, Leprince P, Luyt CE, et al. Outcomes and long-term quality-of-life of patients supported by extracorporeal membrane oxygenation for refractory cardiogenic shock. Crit Care Med 2008;36:1404-11.

22. Shekar K, Fraser JF, Smith MT, et al. Pharmacokinetic changes in patients receiving extracorporeal membrane oxygenation. J Crit Care 2012;27:741.e9-18.

23. Shekar K, Roberts JA, Mcdonald CI, et al. Sequestration

Cite this article as: Franchineau G, Luyt CE, Combes A, Schmidt M. Ventilator-associated pneumonia in extracorporeal membrane oxygenation-assisted patients. Ann Transl Med 2018;6(21):427. doi: 10.21037/atm.2018.10.18 of drugs in the circuit may lead to therapeutic failure during extracorporeal membrane oxygenation. Crit Care 2012;16:R194.

24. Touchard C, Aubry A, Eloy P, et al. Predictors of insufficient peak amikacin concentration in critically ill patients on extracorporeal membrane oxygenation. Critical Care [Internet]. Available online: https://ccforum. biomedcentral.com/articles/10.1186/s13054-018-2122-x

25. Mulla H, McCormack P, Lawson G, et al. Pharmacokinetics of midazolam in neonates undergoing extracorporeal membrane oxygenation. Anesthesiology 2003;99:275-82.

26. Dagan O, Klein J, Gruenwald C, et al. Preliminary studies of the effects of extracorporeal membrane oxygenator on the disposition of common pediatric drugs. Ther Drug Monit 1993;15:263-6.

27. Rosen DA, Rosen KR, Silvasi DL. In vitro variability in fentanyl absorption by different membrane oxygenators. J Cardiothorac Anesth 1990;4:332-5.

28. Fuehner T, Kuehn C, Hadem J, et al. Extracorporeal Membrane Oxygenation in Awake Patients as Bridge to Lung Transplantation. Am J Respir Crit Care Med 2012;185:763-8.

29. Trudzinski FC, Kaestner F, Schäfers HJ, et al. Outcome of Patients with Interstitial Lung Disease Treated with Extracorporeal Membrane Oxygenation for Acute Respiratory Failure. Am J Respir Crit Care Med 2016;193:527-33. 